\title{
On the isotropic constant of random polytopes
}

\author{
David Alonso-Gutiérrez ${ }^{1} \quad$ Alexander E. Litvak ${ }^{2}$ \\ Nicole Tomczak-Jaegermann ${ }^{3}$
}

December 22, 2013

\begin{abstract}
Let $X_{1}, \ldots, X_{N}$ be independent random vectors uniformly distributed on an isotropic convex body $K \subset \mathbb{R}^{N}$, and let $K_{N}$ be the symmetric convex hull of $X_{i}$ 's. We show that with high probability $L_{K_{N}} \leq C \sqrt{\log (2 N / n)}$, where $C$ is an absolute constant. This result closes the gap in known estimates in the range $C n \leq N \leq n^{1+\delta}$. Furthermore, we extend our estimates to the symmetric convex hulls of vectors $y_{1} X_{1}, \ldots, y_{N} X_{N}$, where $y=\left(y_{1}, \ldots, y_{N}\right)$ is a vector in $\mathbb{R}^{N}$.

2010 Classification:

Keywords:
\end{abstract}

\section{Introduction}

In this paper we estimate the isotropic constant of some random polytopes (for the definitions and notations see Section 2). It is known (see e.g. [24]) that among all the convex bodies in $\mathbb{R}^{n}$ the Euclidean ball is the one with the smallest isotropic constant, that is $L_{K} \geq L_{B_{2}^{n}} \geq c$, where $c$ is an absolute positive constant. However, it is still an open problem to determine whether

\footnotetext{
${ }^{1}$ Partially supported by MICINN project MTM2010-16679, MICINN-FEDER project MTM2009-10418, "Programa de Ayudas a Grupos de Excelencia de la Región de Murcia", Fundación Séneca, 04540/GERM/06 and Institut Universitari de Matemàtiques i Aplicacions de Castelló

${ }^{2}$ Research partially supported by the E.W.R. Steacie Memorial Fellowship.

${ }^{3}$ This author holds the Canada Research Chair in Geometric Analysis.
} 
there exists or not an absolute constant $C$ such that for every convex body $K \subset \mathbb{R}^{n}$ one has $L_{K} \leq C$. The boundness of $L_{K}$ by an absolute constant is equivalent to the long standing hyperplane conjecture ([9]). The best general upper bound known up to now is $L_{K} \leq C n^{1 / 4}$ ([18]). This estimate slightly improves (by a logarithmic factor) the earlier Bourgain's upper bound ([10]).

Since remarkable Gluskin's result [14] random polytopes are known to provide many examples of convex bodies (and related normed spaces) with a "patologically bad" behaviour of various parameters of a linear and geometric nature (we refer to the survey [23] and references therein; see also recent examples in [15] and [17]). Not surprisingly, they were also a natural candidate for a potential counterexample for the hyperplane conjecture. This was resolved in [19], where it was shown that the convex hull or the symmetric convex hull of independent Gaussian random vectors in $\mathbb{R}^{n}$ with high probability has the bounded isotropic constant. Some other distributions for vertices were also considered. In all of them the vertices had independent coordinates.

Following the ideas in [19], the problem of estimating of the isotropic constant of random polytopes was considered in [3], for independent random vectors distributed uniformly on the sphere $S^{n-1}$, and in [11], for independent random vectors uniformly distributed on an isotropic unconditional convex body. Also in these cases the isotropic constant of random polytopes generated by these vectors is bounded with high probability. One can check that the same method works for independent random vectors uniformly distributed on a $\psi_{2}$ isotropic convex body as well.

In this paper we estimate the isotropic constant of a random polytope in an isotropic convex body (see Section 2 for the definitions). It is known (see [5], [16] or [4]) that if $K_{N}$ is a polytope in $\mathbb{R}^{n}$ with $N$ vertices then

$$
L_{K_{N}} \leq C \min \left\{\sqrt{\frac{N}{n}}, \log N\right\},
$$

where $C$ is an absolute constant.

In $[12,13]$, the authors provided a lower estimate for the volume of a random polytope $K_{N}$ obtained as the convex hull of $N \leq e^{\sqrt{n}}$ random points, namely

$$
\left|K_{N}\right|^{\frac{1}{n}} \geq c \sqrt{\frac{\log \frac{N}{n}}{n}} L_{K}
$$


(see end of Section 2 for more precise formulation and details). On the other hand, the proof of the estimate $L_{K_{N}} \leq C \log N$ in [4] passes through showing that if $X_{1}, \ldots, X_{N}$ are the vertices of $K_{N}$, then for any affine transformation $T$ we have

$$
L_{K_{N}} \leq \frac{C \max _{1 \leq i \leq N}\left|T X_{i}\right| \log N}{n\left|T K_{N}\right|^{\frac{1}{n}}} .
$$

Consequently, taking $T$ to be the identity operator and using the concentration of measure result proved by Paouris [26], we obtain that if $K_{N}$ is the convex hull or the symmetric convex hull of $n+1 \leq N \leq e^{\sqrt{n}}$ independent random vectors uniformly distributed on an isotropic convex body, then with high probability

$$
L_{K_{N}} \leq \frac{C \log N}{\sqrt{\log \frac{N}{n}}}
$$

Notice that if $N \geq n^{1+\delta}, \delta \in(0,1)$, this estimate does not exceed $(C / \delta) \sqrt{\log \frac{N}{n}}$. However, the constant $C / \delta$ tends to infinity as $\delta$ tends to 0 . On the other hand, if $N$ is proportional to $n$ the isotropic constant of $K_{N}$ is bounded (by an absolute constant), while the upper bound in (1.1) is not. The following theorem closes the the gap between $N \leq c n$ and $N \geq n^{1+\delta}$.

Theorem 1.1. There exist absolute positive constants $c, C$ such that if $n \leq$ $N$, and $X_{1}, \ldots, X_{N}$ are independent random vectors uniformly distributed on an isotropic convex body $K$, and $K_{N}$ is their symmetric convex hull, then

$$
\mathbb{P}\left(\left\{L_{K_{N}} \leq C \sqrt{\log \frac{2 N}{n}}\right\}\right) \geq 1-\exp (-c \sqrt{n}) .
$$

Furthermore, we extend Theorem 1.1 to a much more general setting, namely to a family of perturbations of a random polytope. To desribe our extension we need more notations.

For a vector $y=\left(y_{1}, \ldots, y_{N}\right) \in \mathbb{R}^{N}$, we denote by $y^{*}=\left(y_{1}^{*}, \ldots, y_{N}^{*}\right)$ the vector, whose coordinates are the decreasing rearrangement of $\left\{\left|y_{i}\right|\right\}_{i}$. Given $k \leq N$ we consider the following norm

$$
\|y\|_{k, 2}:=\left(\sum_{i=1}^{k} y_{i}^{* 2}\right)^{1 / 2}=\max \left|P_{E} y\right|
$$


where the maximum is taken over all coordinate subspaces $E$ of dimension $k$. The ball of radius $a$ in this norm we denote by

$$
B(a):=\left\{y \in \mathbb{R}^{N}:\|y\|_{k, 2} \leq a\right\} .
$$

For $n \leq m \leq N$ and $y \in \mathbb{R}^{N}$ denote

$$
\alpha_{y, m}:=\left(\prod_{i=m-n+1}^{m}\left|y_{i}^{*}\right|\right)^{\frac{1}{n}} \geq y_{m}^{*}
$$

Let $X_{1}, \ldots, X_{N} \in \mathbb{R}^{n}$ and $y \in \mathbb{R}^{N}$. Denote

$$
K_{N, y}=\operatorname{conv}\left\{ \pm y_{1} X_{1}, \ldots, \pm y_{N} X_{N}\right\} .
$$

Theorem 1.2. There exist absolute positive constants $c$ and $C$ such that the following holds. Let $C n \leq N$ and let $X_{1}, \ldots, X_{N}$ be independent random vectors uniformly distributed on an isotropic convex body $K$. Then for every $y \in \mathbb{R}^{N}$ the event

$$
L_{K_{N, y}} \leq C \frac{\|y\|_{n, 2}}{\sqrt{n}} \inf \left\{\alpha_{y, m}^{-1} \frac{\log (2 N / n)}{\sqrt{\log (2 m / n)}} \mid C n \leq m \leq N\right\}
$$

occurs with probability greater than $1-e^{-c \sqrt{n}}$. Moreover, for every a $>0$ the event

$$
\sup _{y \in B(a)} L_{K_{N, y}} \leq C \frac{a}{\sqrt{n}} \inf \left\{\alpha_{y, m}^{-1} \frac{\log (2 N / n)}{\sqrt{\log (2 m / n)}} \mid N-\frac{c N}{\log N} \leq m \leq N\right\}
$$

occurs with probability greater than $1-e^{-c \sqrt{n}}$.

Remark 1. Note that if $n \leq N \leq C n$ then $L_{K} \leq C$ for any symmetric polytope $K$ generated by $N$ vectors ([5]).

Remark 2. Clearly Theorem 1.2 applied to the vector $y=(1, \ldots, 1)$ implies Theorem 1.1.

Finally, in the last section, we consider the case when the vector describing the perturbation is also random. Such a setting has been recently considered in [6]. In Theorem 4.2 we show that for the Gaussian vector $y$ in $\mathbb{R}^{N}$, under ceratin conditions on the $\psi_{2}$ behavior of linear functionals on $K$, with high probability we have

$$
L_{K_{N}, y} \leq C \sqrt{\log \frac{2 N}{n}}
$$




\section{Preliminaries}

Along the paper, the letters $c, C, c_{1}, C_{1}, \ldots$ will always denote absolute positive constants, whose values may change from line to line. Given two functions $f$ and $g$ we say that they are equivalent and write $f \approx g$ if $c_{1} f \leq g \leq c_{2} f$.

By $|\cdot|$ and $\langle\cdot, \cdot\rangle$ we denote the canonical Euclidean norm and the canonical inner product on $\mathbb{R}^{n}$. The (unit) Euclidean ball and sphere are denoted by $B_{2}^{n}$ and $S^{n-1}$. Let $K$ be a symmetric convex body in $\mathbb{R}^{n}$ and let $\|\cdot\|_{K}$ be its associated norm

$$
\|x\|_{K}=\inf \{\lambda>0: x \in \lambda K\} .
$$

The support function of $K$ is $h_{K}(y)=\max _{x \in K}\langle x, y\rangle$ and it is the norm associated to the polar body of $K$,

$$
K^{\circ}=\left\{y \in \mathbb{R}^{n}:\langle x, y\rangle \leq 1 \forall x \in K\right\} .
$$

Given convex body $K$ we denote by $|K|$ its volume. We also denote by $|E|$ the cardinality of a finite set $E$. For $E \subset\{1, \ldots, N\}$ the coordinate projection on $\mathbb{R}^{E}$ is denoted by $P_{E}$.

We say that a convex body $K \subseteq \mathbb{R}^{n}$ is isotropic if it has volume $|K|=1$, its center of mass is at 0 (i.e. $\int_{K} x d x=0$ ) and for every $\theta \in S^{n-1}$ one has

$$
\int_{K}\langle x, \theta\rangle^{2} d x=L_{K}^{2},
$$

where $L_{K}$ is a constant independent of $\theta . L_{K}$ is called the isotropic constant of $K$.

It is known that every convex body has a unique (up to an orthogonal transformation) affine image that is isotropic. This allows to define the isotropic constant of any convex body as the isotropic constant of its isotropic image. It is also known (see e.g. [24]) that

$$
n L_{K}^{2}=\inf \left\{\frac{1}{|T K|^{1+\frac{2}{n}}} \int_{a+T K}|x|^{2} d x: T \in G L(n), a \in \mathbb{R}^{n}\right\} .
$$

We need two more results on the distribution of Euclidean norms of random vectors and their sums. Let $X_{i}, i \leq N$, be independent random vectors 
uniformly distributed in an isotropic convex body $K \in \mathbb{R}^{n}$. Let $A$ be a random $n \times N$ matrix, whose columns are $X_{i}$ 's. For $m \leq N$ denote

$$
A_{m}=\sup \left\{|A y|\left|y \in B_{2}^{N},\right| \operatorname{supp} y \mid \leq m\right\}
$$

(supp denotes the support of $y$ ). Theorem 3.13 in [1] (note the different normalization) implies the following estimate.

Theorem 2.1. There is an absolute positive constant $C$ such that for every $\gamma \geq 1$ and every $m \leq N$

$$
\mathbb{P}\left(\left\{A_{m} \geq C L_{K} \gamma \sqrt{m} \log \frac{2 N}{m}+6 \max _{i}\left|X_{i}\right|\right\}\right) \leq \exp \left(-\gamma \sqrt{m} \log \frac{2 N}{m}\right) .
$$

The following theorem is a combination of Paouris' theorem ([26], see also [2] for a short proof) with the union bound (cf. Lemma 3.1 in [1]).

Theorem 2.2. There exists an absolute positive constant $C$ such that for any $N \leq \exp (\sqrt{n})$ and for every $\lambda \geq 1$ one has

$$
\mathbb{P}\left(\left\{\max _{i \leq N}\left|X_{i}\right| \geq C \lambda \sqrt{n} L_{K}\right\}\right) \leq \exp (-\lambda \sqrt{n}) .
$$

Finally we need the estimate on the volume of the random polytope

$$
K_{N}=\operatorname{conv}\left\{ \pm X_{1}, \ldots, \pm X_{N}\right\}
$$

where $X_{i}, i \leq N$, are independent random vectors uniformly distributed in an isotropic convex body $K \subset \mathbb{R}^{n}$. The estimates of the following theorem were observed in [12] (see Fact 3.2, the remarks following it, and Fact 3.3 there).

Theorem 2.3. There are absolute positive constants $C, c_{1}, c_{2}$ such that for $C n \leq N \leq e^{\sqrt{n}}$,

$$
\mathbb{P}\left(\left\{\left|K_{N}\right|^{1 / n} \geq c_{1} \sqrt{\frac{\log (N / n)}{n}} L_{K}\right\}\right) \geq 1-\exp \left(-c_{2} \sqrt{N}\right) .
$$

In fact this theorem is a combination of three results. The first says that $K_{N}$ contains the centroid body. Recall that for $p \geq 1$ the $p$-centroid body 
$Z_{p}(K)$ was introduced in [22] (with a different normalization) as the convex body, whose support function is

$$
h_{Z_{p}(K)}(\theta)=\left(\int_{K}|\langle x, \theta\rangle|^{p} d x\right)^{\frac{1}{p}} .
$$

In [13] (Theorem 1.1) the authors proved that for every parameters $\beta \in$ $(0,1 / 2)$ and $\gamma>1$ one has the inclusion $K_{N} \supset c_{1} Z_{p}(K)$ for $p=c_{2} \beta \log (2 N / n)$ and $N \geq c_{3} \gamma n$ with the probability greater than

$$
1-\exp \left(-c_{4} N^{1-\beta} n^{\beta}\right)-\mathbb{P}\left(\left\{\|A\|>\gamma L_{K} \sqrt{N}\right\}\right),
$$

where $A$ is the random matrix whose columns are $X_{1}, \ldots, X_{N}$. The probability that norm of $A$ (note $\|A\|=A_{N}$ ) is large was estimated in [1] (combine Theorems 2.1 and 2.2 above). Finally, from results of [20] and [26] the bound

$$
\left|Z_{p}(K)\right|^{1 / n} \approx \sqrt{p / n} L_{K}
$$

more details?

follows provided that $p \leq \sqrt{n}$ (it improves the bound provided in [21]).

We also will need the definition of $\psi_{\alpha}$ norm. For a real random variable $z$ and $\alpha \in[1,2]$ we define the $\psi_{\alpha}$-norm by

$$
\|z\|_{\psi_{\alpha}}=\inf \left\{C>0 \mid \mathbb{E} \exp (|Y| / C)^{\alpha} \leq 2\right\} .
$$

It is well known that the the condition $\|z\|_{\psi_{\alpha}} \leq c_{1}$ is equivalent to the condition

$$
\forall p>1: \quad\left(\mathbb{E}|z|^{p}\right)^{1 / p} \leq c_{2} p^{1 / \alpha} \mathbb{E}|z| .
$$

Let $X$ be a centered random vector in $\mathbb{R}^{n}$ and $\alpha>0$. We say that $X$ is $\psi_{\alpha}$ or a $\psi_{\alpha}$ vector, if

$$
\|X\|_{\psi_{\alpha}}:=\sup _{y \in S^{n-1}}\|\langle X, y\rangle\|_{\psi_{\alpha}}<\infty
$$

\section{Proofs}

In this section we prove Theorem 1.2. The proof consists of two propositions. 
Proposition 3.1. Let $n \leq N \leq e^{\sqrt{n}}$ and $X_{1}, \ldots, X_{N}$ are independent random vectors distributed uniformly on an isotropic convex body K. Let a $>0$. Then the event

$$
\sup \left\{\frac{1}{\left|K_{N, y}\right|} \int_{K_{N, y}}|x|^{2} d x \quad \mid y \in B(a), y_{n}^{*}>0\right\} \leq C \frac{a^{2}}{n} L_{K}^{2} \log ^{2} \frac{2 N}{n}
$$

occurs with probability greater than $1-\exp (-\sqrt{n} \log (2 N / n))$, where $C$ is an absolute constant.

To prove this proposition we need the following lemma.

Lemma 3.2. Let $1 \leq n \leq N$ be integers and $P=\operatorname{conv}\left\{P_{1}, \ldots, P_{N}\right\}$ be a non-degenerated symmetric polytope in $\mathbb{R}^{n}$. Then

$$
\frac{1}{|P|} \int_{P}|x|^{2} d x \leq \frac{1}{(n+1)(n+2)} \sup _{E}\left(\sum_{i \in E}\left|P_{i}\right|^{2}+\left|\sum_{i \in E} P_{i}\right|^{2}\right),
$$

where the supremum is taken over all subsets $E \subset\{1, \ldots, N\}$ of cardinality $n$.

Proof. We can decompose $P$ as a disjoint union of simplices (up to sets of measure 0 ), say $P=\cup_{i=1}^{\ell} C_{i}$, where each $C_{i}$ is of the form $\operatorname{conv}\left\{0, P_{i_{1}}, \ldots, P_{i_{n}}\right\}$ for some choice of $P_{i_{j}}$ 's. For every such $C_{i}$, denote $F_{i}:=\operatorname{conv}\left\{P_{i_{1}}, \ldots, P_{i_{n}}\right\}$. Then for any integrable function $f$ we have

$\int_{C_{i}} f(x) d x=\int_{F_{i}} \int_{0}^{1} r^{n-1} f(r y)|\langle y, \nu(y)\rangle| d y d r=d\left(0, F_{i}\right) \int_{F_{i}} \int_{0}^{1} r^{n-1} f(r y) d y d r$,

where $\nu(y)$ is the outer normal vector to $P$ at the point $y$ and $d\left(0, F_{i}\right)$ is the distance from the origin to the affine subspace spanned by $F_{i}$. Thus, as in [19], for every $i \leq \ell$ one has

$$
\left|C_{i}\right|=n^{-1}\left|F_{i}\right| d\left(0, F_{i}\right) \quad \text { and } \quad \int_{C_{i}}|x|^{2}=\frac{d\left(0, F_{i}\right)}{n+2} \int_{F_{i}}|y|^{2} d y .
$$

In particular,

$$
|P|=\sum_{i=1}^{\ell}\left|C_{i}\right|=\frac{1}{n} \sum_{i=1}^{\ell}\left|F_{i}\right| d\left(0, F_{i}\right)
$$


Therefore,

$$
\begin{aligned}
\frac{1}{|P|} \int_{P}|x|^{2} d x & =\frac{1}{|P|} \sum_{i=1}^{\ell} \frac{d\left(0, F_{i}\right)}{n+2} \int_{F_{i}}|y|^{2} d y \\
& \leq \frac{1}{|P|} \sum_{i=1}^{\ell} \frac{d\left(0, F_{i}\right)\left|F_{i}\right|}{n+2} \sup _{1 \leq i \leq \ell} \frac{1}{\left|F_{i}\right|} \int_{F_{i}}|y|^{2} d y \\
& \leq \frac{n}{n+2} \sup _{F} \frac{1}{|F|} \int_{F}|y|^{2} d y
\end{aligned}
$$

where the supremum is taken over all $F=\operatorname{conv}\left\{P_{i_{1}}, \ldots, P_{i_{n}}\right\}$. Note that any such $F$ can be presented as $F=T \Delta^{n-1}$, where $\Delta^{n-1}=\operatorname{conv}\left\{e_{1}, \ldots, e_{n}\right\}$ denotes the regular $n-1$ dimensional simplex in $\mathbb{R}^{n}$ and $T$ is the matrix whose columns are the vectors $P_{i_{j}}$. Since

$$
\frac{1}{\left|\Delta^{n-1}\right|} \int_{\Delta^{n-1}} y_{i} y_{j} d y=\frac{1+\delta_{i j}}{n(n+1)},
$$

where $\delta_{i j}$ is the Kronecker delta, for every $F=\operatorname{conv}\left\{P_{i_{1}}, \ldots, P_{i_{n}}\right\}$ we obtain

$$
\frac{1}{|F|} \int_{F}|y|^{2} d y=\frac{1}{n(n+1)}\left(\sum_{j=1}^{n}\left|P_{i_{j}}\right|^{2}+\left|\sum_{j=1}^{n} P_{i_{j}}\right|^{2}\right) .
$$

This implies the desire estimate.

Proof of Proposition 3.1. Note that if $y_{N}^{*}>0$ then the cardinality of support of $y$ is at least $n$, so $K_{N, y}$ is not degenerated with probability one. Therefore, with probability one $K_{N, y}$ is non-degenerated for any countable dense set in $B_{0}(a):=\left\{y \in B(a) \mid y_{n}^{*}>0\right\}$. Clearly, the supremum under question is the same over $y \in B_{0}(a)$ and over such a dense set.

Now, by Lemma 3.2 we have that $\sup _{y \in B_{0}(a)}\left|K_{N, y}\right|^{-1} \int_{K_{N, y}}|x|^{2} d x$ is bounded from above by

$$
\frac{1}{(n+1)(n+2)} \sup _{y \in B_{0}(a)} \sup _{|E|=n}\left(\sum_{i \in E}\left|y_{i} X_{i}\right|^{2}+\left|\sum_{i \in E} y_{i} X_{i}\right|^{2}\right)
$$

(formally, we should additionally take supremum over $\varepsilon_{i}= \pm 1$ and to have $y_{i} \varepsilon_{i} X_{i}$ in the formula under suprema, but, since $B_{0}(a)$ is unconditional, the supremum over $\varepsilon_{i}$ 's can be omitted). 
Note that

$$
\sum_{i \in E}\left|y_{i} X_{i}\right|^{2} \leq\|y\|_{n, 2}^{2} \max _{i \leq N}\left|X_{i}\right|^{2}
$$

and

$$
\left|\sum_{i \in E} y_{i} X_{i}\right|=\left|A P_{E} y\right| \leq A_{n}\|y\|_{n, 2},
$$

where $A$ is the matrix whose columns are $X_{1}, \ldots, X_{N}$. Therefore, applying Theorem 2.1 and Theorem 2.2 (with $m=n$ and $\lambda=2 \log (2 N / n)$ ) we obtain that

$$
\left.\sup _{y \in B_{0}(a)|E|=n} \sup _{|E|=E}\left|\sum_{i} X_{i}\right|^{2}+\left|\sum_{i \in E} y_{i} X_{i}\right|^{2}\right) \leq a^{2} n L_{K}^{2} \log ^{2} \frac{2 N}{n}
$$

with probability greater than $1-\exp (-\sqrt{n} \log (2 N / n))$.

Proposition 3.3. There exist absolute positive constants $c_{1}, c_{2}, C$ such that if $C n \leq N \leq e^{\sqrt{n}}$ and $X_{1}, \ldots, X_{N}$ are independent random vectors distributed uniformly on an isotropic convex body $K$, then for every $y \in \mathbb{R}^{N}$,

$\mathbb{P}\left(\left\{\forall m \geq C n: \quad\left|K_{N, y}\right|^{\frac{1}{n}} \geq c_{1} \alpha_{y, m} L_{K} \sqrt{\frac{\log (2 m / n)}{n}}\right)\right\} \geq 1-\exp \left(-c_{2} \sqrt{n}\right)$.

Moreover, the event

$$
\forall m \geq N-\frac{c_{1} N}{\log N} \forall y \in \mathbb{R}^{N} \quad\left|K_{N, y}\right|^{\frac{1}{n}} \geq c_{1} \alpha_{y, m} L_{K} \sqrt{\frac{\log (2 m / n)}{n}}
$$

occurs with probability greater than $1-\exp \left(-c_{2} \sqrt{N}\right)$.

The probability estimates in Proposition 3.3 are based on an estimate of corresponding probability for a fixed $y$ and the union bound. We start with the following lemma.

Lemma 3.4. There exist absolute positive constants $c_{1}, c_{2}, C$ such that the following holds. Let $C n \leq m \leq N \leq e^{\sqrt{n}}$ and $X_{1}, \ldots, X_{N}$ are independent random vectors distributed uniformly on an isotropic convex body $K$. Then for every $y \in \mathbb{R}^{N}$ with $y_{m}^{*}>0$ there exists $v=v(y) \in \mathbb{R}^{N}$ having $0 / 1$ coordinates with exactly $m$ ones such that

$$
\left|K_{N, y}\right| \geq \alpha_{y, m}\left|K_{N, v}\right|
$$


and

$$
\mathbb{P}\left(\left\{\left|K_{N, v}\right|^{\frac{1}{n}} \geq c_{1} L_{K} \sqrt{\frac{\log (2 m / n)}{n}}\right\}\right) \geq 1-\exp \left(-c_{2} \sqrt{m}\right) .
$$

Proof. Fix $y \in \mathbb{R}^{N}$ with $y_{m}^{*}>0$ (i.e. $|\operatorname{supp} y| \geq m$ ). Let $i_{1}, \ldots, i_{m}$ be the indices such that $y_{i_{j}}=y_{j}^{*}$ and let $v=v(y) \in \mathbb{R}^{N}$ be the vector with $v_{k}=1$ if $k=i_{j}$ and 0 otherwise. Decompose the polytope $K_{N, v}$ into a disjoint union of simplices (up to a set of zero measure)

$$
K_{N, v}=\bigcup_{k=1}^{\ell} C_{k}
$$

where $C_{k}=\operatorname{conv}\left\{0, \varepsilon_{k_{1}} X_{k_{1}}, \ldots, \varepsilon_{k_{n}} X_{k_{n}}\right\}$ for some $\varepsilon_{k_{j}}= \pm 1$ and some vectors $X_{k_{j}}$, given by the simplicial decomposition of the facets of $K_{N, v}$. Denote

$$
C_{k, y}=\operatorname{conv}\left\{0, \varepsilon_{k_{1}}\left|y_{k_{1}}\right| X_{k_{1}}, \ldots, \varepsilon_{k_{n}}\left|y_{k_{n}}\right| X_{k_{n}}\right\} \subset K_{N, y} .
$$

Clearly, $C_{k, y}$ 's are also disjoint up to a set of zero measure and

$$
\begin{aligned}
\left|C_{k}\right| & =\left|\operatorname{det}\left(\varepsilon_{k_{1}} X_{k_{1}}, \ldots, \varepsilon_{k_{n}} X_{k_{n}}|| \operatorname{conv}\left\{0, e_{1}, \ldots, e_{n}\right\}\right)\right| \\
& \leq \frac{1}{\alpha_{y, m}}\left|\operatorname{det}\left(\varepsilon_{k_{1}}\left|y_{k_{1}}\right| X_{k_{1}}, \ldots, \varepsilon_{k_{n}}\left|y_{k_{n}}\right| X_{k_{n}}\right)\right|\left|\operatorname{conv}\left\{0, e_{1}, \ldots, e_{n}\right\}\right| .
\end{aligned}
$$

This implies

$$
\left|K_{N, v}\right|=\sum_{k=1}^{\ell} C_{k} \leq \alpha_{y, m}^{-1} \sum_{k=1}^{\ell} C_{k, y} \leq \alpha_{y, m}^{-1}\left|K_{N, y}\right| .
$$

This proves the first estimate. The second one follows by Theorem 2.3, since $K_{N, v}$ is a symmetric random polytope in an isotropic convex body generated by $m \geq C n$ random points.

Proof of Proposition 3.3. Without loss of generality we only consider $y$ 's satisfying $y_{n}^{*}>0$ (otherwise estimates are trivial).

The first estimate follows from Lemma 3.4 and the union bound, since

$$
\sum_{m \geq C n} e^{-c_{2} \sqrt{m}} \leq e^{-c_{2} \sqrt{n}}
$$


provided that $C$ is large enough.

To prove the second bound note that the set $\{v(y)\}_{y \in \mathbb{R}^{N}}(v(y)$ is from Lemma 3.4) has cadinality $\left(\begin{array}{l}N \\ m\end{array}\right)$ and that denoting $k=N-m$

$$
\left(\begin{array}{l}
N \\
m
\end{array}\right) \exp \left(-c_{2} \sqrt{m}\right) \leq \exp \left(-c_{2} \sqrt{m}+k \log (e N / k)\right) \leq \exp \left(-c_{2} \sqrt{m} / 2\right),
$$

provided that $k \leq c \sqrt{N} / \log N$. Lemma 3.4 and the union bound imply

$\mathbb{P}\left(\left\{\forall y \in \mathbb{R}^{N}:\left|K_{N, v}\right|^{\frac{1}{n}} \geq c_{1} \alpha_{y, m} L_{K} \sqrt{\frac{\log (2 m / n)}{n}}\right\}\right) \geq 1-\exp \left(-c_{2} \sqrt{m} / 2\right)$.

The result follows by the union bound and (3.1).

Proof of Theorem 1.2. For $C n \leq N \leq e^{\sqrt{N}}$ Propositions 3.1 and 3.3 imply the result, since, by (2.1),

$$
n L_{K_{N, y}}^{2} \leq \frac{1}{\left|K_{N, y}\right|^{1+\frac{2}{n}}} \int_{K_{N, y}}|x|^{2} d x .
$$

For $N \geq e^{\sqrt{n}}$ the theorem follows from the general estimate $L_{K} \leq C n^{1 / 4}$ for any $n$-dimensional convex body ([18]).

\section{Random perturbations of random polytopes}

Recall that a $Z_{p}$ body and $\psi_{\alpha}$ norm were defined in (2.2) and (2.4). In this section $G=\left(g_{1}, \ldots, g_{N}\right)$ denotes a standard Gaussian random vector in $\mathbb{R}^{N}$, independent of any other random variables. We also denote

$$
\gamma_{p}:=\left(\mathbb{E}\left|g_{1}\right|^{p}\right)^{1 / p} \approx \sqrt{p}
$$

Proposition 4.1. There are absolute positive constants $C, c_{1}$ and $c_{2}$ such that the following holds. Let $\beta>2$ and $N \geq C(\log \beta)^{2} n$. Let $X_{1}, \ldots, X_{N}$ be independent copies of a random vector uniformly distributed on an isotropic convex body $K$. Assume that $\left\|X_{1}\right\|_{\psi_{2}} \leq \beta L_{K} \sqrt{\log \frac{N}{n}}$. Then

$$
\mathbb{P}_{G, X_{1}, \ldots, X_{N}}\left(\left\{K_{N, G} \supseteq c_{1} \sqrt{\log \frac{N}{n}} Z_{\log (N / n)}(K)\right\}\right) \geq 1-\exp \left(-c_{2} \sqrt{N}\right) .
$$


Proof. First note that for any $p \geq 1, i \leq N$ and $\theta \in S^{n-1}$, one has

$$
\left(\mathbb{E}\left|\left\langle g_{i} X_{i}, \theta\right\rangle\right|^{p}\right)^{1 / p}=\left(\mathbb{E}\left|g_{i}\right|^{p}\right)^{1 / p}\left(\mathbb{E}\left|\left\langle X_{i}, \theta\right\rangle\right|^{p}\right)^{1 / p} \leq c_{1} \sqrt{p}\left(\mathbb{E}\left|\left\langle X_{i}, \theta\right\rangle\right|^{p}\right)^{1 / p} .
$$

Thus,

$$
\sup _{i \leq N} \sup _{\theta \in S^{n-1}}\left\|\left\langle g_{i} X_{i}, \theta\right\rangle\right\|_{\psi_{1}} \leq c_{2} \sup _{i \leq N} \sup _{\theta \in S^{n-1}}\left\|\left\langle X_{i}, \theta\right\rangle\right\|_{\psi_{2}} \leq c_{3} \beta L_{K} \sqrt{\log \frac{N}{n}} .
$$

Denote by $A$ the $n \times N$ random matrix whose columns are the vectors $g_{i} X_{i}$. By Theorem 3.13 in [1] (cf. Theorem 2.1),

$$
\mathbb{P}_{G, X_{1}, \ldots, X_{N}}\left(\left\{\|A\| \geq c_{4} \beta L_{K} \sqrt{N \log \frac{N}{n}}+6 \max _{i \leq N}\left|X_{i}\right|\right\}\right) \leq \exp (-2 \sqrt{N}) .
$$

Together with Theorem 2.2 (applied with $\lambda=2 \sqrt{n}$ ), we have that

$$
\mathbb{P}_{G, X_{1}, \ldots, X_{N}}\left(\left\{\|A\| \geq c_{5} \beta L_{K} \sqrt{N \log \frac{N}{n}}\right\}\right) \leq e^{-\sqrt{N}}
$$

On the other hand, for every $\sigma \subseteq\{1, \ldots, N\}, q \geq 1$ and $\theta \in S^{n-1}$, by Paley-Zygmund inequality,

$$
\begin{aligned}
& \mathbb{P}_{G, X_{1}, \ldots, X_{N}}\left(\left\{\max _{i \in \sigma}\left|\left\langle g_{i} X_{i}, \theta\right\rangle\right| \leq \frac{1}{2}\left(\mathbb{E}\left|g_{1}\right|^{q}\right)^{\frac{1}{q}}\left(\mathbb{E}\left|\left\langle X_{1}, \theta\right\rangle\right|^{q}\right)^{\frac{1}{q}}\right\}\right) \\
= & \prod_{i \in \sigma} \mathbb{P}_{G, X_{1}, \ldots, X_{N}}\left(\left\{\left|\left\langle g_{i} X_{i}, \theta\right\rangle\right| \leq \frac{1}{2}\left(\mathbb{E}\left|g_{1}\right|^{q}\right)^{\frac{1}{q}}\left(\mathbb{E}\left|\left\langle X_{1}, \theta\right\rangle\right|^{q}\right)^{\frac{1}{q}}\right\}\right) \\
\leq & \left(1-\left(1-\left(\frac{1}{2}\right)^{q}\right)^{2} \frac{\left(\mathbb{E}\left|g_{1}\right|^{q} \mathbb{E}\left|\left\langle X_{1}, \theta\right\rangle\right|^{q}\right)^{2}}{\mathbb{E}\left|g_{1}\right|^{2 q} \mathbb{E}\left|\left\langle X_{1}, \theta\right\rangle\right|^{2 q}}\right)^{|\sigma|} .
\end{aligned}
$$

Since $\gamma_{p} \approx \sqrt{p}$, and from Borell's lemma ([8], see also Appendix III in [25]),

$$
\mathbb{E}\left|\left\langle X_{1}, \theta\right\rangle\right|^{2 q} \leq c_{6}^{q} \mathbb{E}\left|\left\langle X_{1}, \theta\right\rangle\right|^{q},
$$

the quantity above is bounded by

$$
\left(1-\frac{1}{4 C^{q}}\right)^{|\sigma|} \leq \exp \left(-\frac{|\sigma|}{4 C^{q}}\right)
$$


Set $m=[N / n]$. Let $\sigma_{1}, \ldots, \sigma_{n}$ be a partition of $\{1, \ldots, N\}$ with $m \leq\left|\sigma_{i}\right|$ for every $i$ and $\|\cdot\|_{0}$ be the norm

$$
\|u\|_{0}=\frac{1}{n} \sum_{i=1}^{n} \max _{j \in \sigma_{i}}\left|u_{j}\right| .
$$

Note that $\|\cdot\| \leq n^{-1 / 2}|\cdot|$. Since for all $1 \leq i \leq k$ and every $z \in \mathbb{R}^{n}$

$$
h_{K_{N, G}}(z)=\max _{1 \leq j \leq N}\left|\left\langle g_{j} X_{j}, z\right\rangle\right| \geq \max _{j \in \sigma_{i}}\left|\left\langle g_{j} X_{j}, z\right\rangle\right|,
$$

then for every $z \in \mathbb{R}^{n}$

$$
h_{K_{N, G}}(z) \geq\|A z\|_{0} .
$$

Cleary, if $z \in \mathbb{R}^{n}$ verifies $\|A z\|_{0} \leq \frac{1}{4} \gamma_{q}\left(\mathbb{E}\left|\left\langle X_{1}, z\right\rangle\right|^{q}\right)^{\frac{1}{q}}$, then there exists a set $I \subseteq\{1, \ldots, k\}$ with $|I| \geq \frac{k}{2}$ such that

$$
\max _{j \in \sigma_{i}}\left|\left\langle g_{j} X_{j}, z\right\rangle\right| \leq \frac{1}{2} \gamma_{q}\left(\mathbb{E}\left|\left\langle X_{1}, z\right\rangle\right|^{q}\right)^{\frac{1}{q}}
$$

for every $i \in I$. Thus, for every $z \in \mathbb{R}^{n}$,

$$
\begin{aligned}
& \mathbb{P}_{G, X_{1}, \ldots, X_{N}}\left(\left\{\|A z\|_{0} \leq \frac{1}{4} \gamma_{q}\left(\mathbb{E}\left|\left\langle X_{1}, z\right\rangle\right|^{q}\right)^{\frac{1}{q}}\right\}\right) \\
\leq & \sum_{|I|=\left\lceil\frac{n}{2}\right\rceil} \mathbb{P}_{G, X_{1}, \ldots, X_{N}}\left(\left\{\forall i \in I: \max _{j \in \sigma_{i}}\left|\left\langle g_{j} X_{j}, z\right\rangle\right| \leq \frac{1}{2} \gamma_{q}\left(\mathbb{E}\left|\left\langle X_{1}, z\right\rangle\right|^{q}\right)^{\frac{1}{q}}\right\}\right) \\
\leq & \sum_{|I|=\left\lceil\frac{n}{2}\right\rceil} \prod_{i \in I} \mathbb{P}_{G, X_{1}, \ldots, X_{N}}\left(\left\{\max _{j \in \sigma_{i}}\left|\left\langle g_{j} X_{j}, z\right\rangle\right| \leq \frac{1}{2} \gamma_{q}\left(\mathbb{E}\left|\left\langle X_{1}, z\right\rangle\right|^{q}\right)^{\frac{1}{q}}\right\}\right) \\
\leq & \sum_{|I|=\left\lceil\frac{n}{2}\right\rceil} \prod_{i \in I} \exp \left(-\frac{\left|\sigma_{i}\right|}{4 C^{q}}\right) \leq 2^{n} \exp \left(-\frac{n m}{4 C^{q}}\right) \leq 2^{n} \exp \left(-\frac{N}{8 C^{q}}\right) \\
\leq & \exp \left(-\frac{\sqrt{N n}}{16}\right)
\end{aligned}
$$

provided that $q:=(1 / 2) \log (N / n)$ and $N>125 n$.

Now, let

$$
S=\left\{z \in \mathbb{R}^{n} \mid \frac{1}{2} \gamma_{q}\left(\mathbb{E}\left|\left\langle X_{1}, z\right\rangle\right|^{q}\right)^{\frac{1}{q}}=1\right\}
$$


and let $U \subset S$ be a $\delta$-net (in metric defined by $S$ ) with cardinality $|U| \leq\left(\frac{3}{\delta}\right)^{n}$, i.e., for every $z \in S$ there is $u \in U$ such that $\frac{1}{2} \gamma_{q}\left(\mathbb{E}\left|\left\langle X_{1}, z-u\right\rangle\right|^{q}\right)^{\frac{1}{q}} \leq \delta$. Then

$$
\mathbb{P}\left(\left\{\exists u \in U:\|A u\|_{0} \leq \frac{1}{2}\right\}\right) \leq \exp \left(n \log \frac{3}{\delta}-\sqrt{N n} / 16\right) .
$$

By isotropicity we have that $\left(\mathbb{E}\left|\left\langle X_{1}, z\right\rangle\right|^{q}\right)^{\frac{1}{q}} \geq L_{k}|z|$ (because we have chosen $q=(1 / 2) \log (N / n)>2)$. Thus, assuming $\|A\| \leq c_{5} \beta L_{K} \sqrt{N \log \frac{N}{n}}$, we have

$$
\begin{aligned}
\|A z\|_{0} \leq \frac{1}{\sqrt{n}}|A z| & \leq c_{5} \beta L_{K} \sqrt{\frac{N}{n}} \sqrt{\log \frac{N}{n}}|z| \leq c_{5} \beta \sqrt{\frac{N}{n}} \sqrt{2 q}\left(\mathbb{E}\left|\left\langle X_{1}, z\right\rangle\right|^{q}\right)^{\frac{1}{q}} \\
& \leq c_{6} \beta \gamma_{q} \sqrt{\frac{N}{n}}\left(\mathbb{E}\left|\left\langle X_{1}, z\right\rangle\right|^{q}\right)^{\frac{1}{q}} .
\end{aligned}
$$

Therefore, if $u \in U$ approximates $z \in S$, that is if $\frac{1}{2} \gamma_{q}\left(\mathbb{E}\left|\left\langle X_{1}, z-u\right\rangle\right|^{q}\right)^{\frac{1}{q}} \leq \delta$, then $u$ also satisfies

$$
\|A u\|_{0} \leq\|A z\|_{0}+c_{7} \beta \sqrt{\frac{N}{n}} \delta
$$

Choosing $\delta=\sqrt{n} /\left(4 \beta c_{7} \sqrt{N}\right)$ and denoting the event

$$
\Omega_{0}:=\left\{\|A\| \leq c_{5} \beta L_{K} \sqrt{N \log \frac{N}{n}}\right\}
$$

we obtain

$$
\begin{aligned}
& \mathbb{P}_{G, X_{1}, \ldots, X_{N}}\left(\left\{\omega \in \Omega_{0} \mid \exists z \in \mathbb{R}^{n}:\|A z\|_{0} \leq \frac{1}{8} \gamma_{q}\left(\mathbb{E}\left|\left\langle X_{1}, z\right\rangle\right|^{q}\right)^{\frac{1}{q}}\right\}\right) \\
= & \mathbb{P}_{G, X_{1}, \ldots, X_{N}}\left(\left\{\omega \in \Omega_{0} \mid \exists z \in S:\|A z\|_{0} \leq \frac{1}{4}\right\}\right) \\
\leq & \mathbb{P}_{G, X_{1}, \ldots, X_{N}}\left(\left\{\omega \in \Omega_{0} \mid \exists u \in U:\|A u\|_{0} \leq \frac{1}{2}\right\}\right) \\
\leq & \exp \left(n \log \frac{12 c_{7} \beta \sqrt{N}}{\sqrt{n}}-\sqrt{N n} / 16\right) \leq \exp (-\sqrt{N n} / 20)
\end{aligned}
$$

provided $N \geq C(\log \beta)^{2} n$ for a big enough absolute constant $C$. Since $h_{K_{N, G}}(z)=\|A z\|_{\infty} \geq\|A z\|_{0}$, this together with (4.1) and the definition (2.2), 
implies that with probability at least $1-\exp (-\sqrt{N})-\exp (-\sqrt{N n} / 20)$,

$$
K_{N, G} \supseteq \frac{1}{8} \gamma_{q} Z_{q}(K) \supseteq c \sqrt{\log \frac{N}{n}} Z_{\log (N / n)}(K) .
$$

Proposition 4.1 implies the following theorem.

Theorem 4.2. There are absolute positive constants $C, c_{1}$ and $c_{2}$ such that the following holds. Let $\beta>2$ and $N \geq C(\log \beta)^{2} n$. Let $X_{1}, \ldots, X_{N}$ be independent copies of a random vector uniformly distributed on an isotropic convex body and assume that $\left\|X_{1}\right\|_{\psi_{2}} \leq \beta \sqrt{\log \frac{N}{n}}$. Then

$$
\mathbb{P}_{G, X_{1}, \ldots, X_{N}}\left(\left\{L_{K_{N, G}} \leq c_{1} \sqrt{\log \frac{2 N}{n}}\right\}\right) \geq 1-\exp \left(-c_{2} \sqrt{n} \log \frac{2 N}{n}\right) .
$$

Proof. By Proposition 3.1, the probability (with respect to $X_{i}$ 's) of the event

$$
\forall y \in \mathbb{R}^{n} \text { with } y_{n}^{*}>0: \frac{1}{\left|K_{N, y}\right|} \int_{K_{N, y}}|x|^{2} d x \leq C L_{K}^{2} \frac{\|y\|_{n, 2}^{2}}{n} \log ^{2} \frac{2 N}{n}
$$

is at least $1-\exp (-\sqrt{n} \log (2 N / n))$.

It is well know (and can be directly calculated) that for the Gaussian vector $G=\left(g_{1}, \ldots, g_{N}\right)$ one has

$$
\mathbb{E}\|G\|_{n, 2} \approx \sqrt{n \log \frac{N}{n}}
$$

Using concentration (see e.g. Theorem 1.5 in [27]), we observe that for some absolute constant $C_{1}>0$,

$$
\mathbb{P}_{G}\left(\|G\|_{n, 2} \geq C_{1} \sqrt{n \log \frac{N}{n}}\right) \leq \exp (-n \log (N / n)) .
$$

Therefore, the probability (with respect to $G$ and $X_{i}$ 's) of the event

$$
\frac{1}{\left|K_{N, G}\right|} \int_{K_{N, G}}|x|^{2} d x \leq C L_{K}^{2} \log ^{3} \frac{N}{n}
$$


is at least $1-\exp (-c \sqrt{n} \log (N / n))$. On the other hand by the previous proposition and (2.3)

$$
\mathbb{P}_{G, X_{1}, \ldots, X_{N}}\left(\left\{\left|K_{N, G}\right|^{1 / n} \geq \frac{c L_{K} \log (N / n)}{\sqrt{n}}\right\}\right) \geq 1-\exp \left(-c^{\prime} \sqrt{N}\right) .
$$

Since

$$
n L_{K_{N, G}}^{2} \leq \frac{1}{\left|K_{N, G}\right|^{1+\frac{2}{n}}} \int_{K_{N, G}}|x|^{2} d x
$$

we obtain the desired result.

Remark 4.3. Finally we would like to note that for $N \geq n^{2}$ with high probability $\left|K_{N, G}\right|^{1 / n} \geq\left(c L_{K} \log N\right) / \sqrt{n}$, even if the convex body $K$ is not $\psi_{2}$ (see Lemma 4 in [6]). Therefore in this case, using

$$
\max _{i \leq N}\left|g_{i} X_{i}\right|=\max _{i \leq N}\left|g_{i}\right| \max _{i \leq N}\left|X_{i}\right| \leq C_{1} \sqrt{\log N} \sqrt{n}
$$

with high probability one has

$$
L_{K_{N, G}} \leq c \frac{\max _{i \leq N}\left|g_{i} X_{i}\right| \log N}{n\left|K_{N, G}\right|^{\frac{1}{n}}} \leq C \sqrt{\log N} .
$$

\section{Acknowledgements}

Part of this work was done while the first named author was a postdoctoral fellows at the Department of Mathematical and Statistical Sciences at University of Alberta and continued while he was a "Juan de la Cierva" postdoctoral researcher at the Mathematics Department in Universidad de Murcia. He would like to thank both departments for providing support, good environment and working conditions.

\section{References}

[1] Adamczak R., Litvak A.E., Pajor A., Tomczak-Jaegermann N. Quantitative estimates of the convergence of the empirical covariance matrix in log-concave ensembles. J. Amer. Math. Soc. 23 (2010), no. 2, 535-561.

[2] Adamczak R., Latala R., Litvak A.E., Oleszkiewicz K., Pajor A., Tomczak-Jaegermann N. A short proof of Paouris' inequality, Canadian Math. Bull, to appear. 
[3] Alonso-Gutiérrez D. On the isotropy constant of random convex sets. Proceedings of the AMS. 136 (2008), no. 9, 3293-3300.

[4] Alonso-Gutiérrez D. A remark on the isotropy constant of polytopes. Proceedings of the AMS 139 (2011) no. 7, 2565-2569.

[5] Alonso-Gutiérrez D., Bastero J., Bernués J., Wolff P. On the isotropy constant of projections of polytopes. Journal of Functional Analysis 258 (2010), no. $5,1452-1465$.

[6] Alonso-Gutiérrez D., Prochno J. Mean width of random perturbations of random polytopes, preprint.

[7] BALL K. Volumes of sections of cubes and related problems. GAFA, Lecture Notes in mathematics 1376 (1989), 251-260, Springer-Verlag.

[8] Borell C. Convex measures on locally convex spaces. Ark. Mat. 12 (1974), 239-252.

[9] Bourgain J. On high-dimensional maximal functions associated to convex bodies. Amer. J. Math 108 (1986), no.6, 1467-1476.

[10] Bourgain J. On the distribution of polynomials on high-dimensional convex sets. GAFA (1989-90), Lecture Notes in Math. 1469 (1991), 127-137, Springer, Berlin.

[11] Dafnis N., Giannopoulos A., GuÉdon O. On the isotropic constant of random polytopes. Advances in Geometry 10 (2010), 311-321.

[12] Dafnis N., Giannopoulos A., Tsolomitis A. Quermass integrals and asymptotic shape of random polytopes in an isotropic convex body, Michigan Math. J. 62 (2013), 59-79.

[13] Dafnis N., Giannopoulos A., Tsolomitis A. Asymptotic shape of a random polytope in a convex body, J. Funct. Analysis 257 (2009), 2820-2839.

[14] GLuskin E.D. The diameter of Minkowski compactum roughly equals to $n$. Funktsional. Anal. i Prilozhen. 15 (1981), no. 1, 72-73. English translation: Functional Anal. Appl. 15 (1981), no. 1, 57-58.

[15] Gluskin E.D., Litvak A.E. A remark on vertex index of the convex bodies, GAFA, Lecture Notes in Math., 2050 (2012), 255-265, Springer, Berlin.

[16] Junge M. Hyperplane conjecture for quotient spaces of $L_{p}$., Forum Math. 6 (1994), 617-635.

[17] Litvak A.E., Rudelson M., Tomczak-Jaegermann N. On approximation by projections of polytopes with few facets, Isr. J. Math., to appear.

[18] Klartag B. On convex perturbations with a bounded isotropic constant. Geom. and Funct. Anal. (GAFA), 16 (2006), no. 6, 1274-1290.

[19] Klartag B., Kozma G. On the hyperplane conjecture for random convex sets, Israel J. Math. 170 (2009), no. 1, 1-33.

[20] Klartag B., Milman E. Centroid bodies and the logarithmic Laplace transform a unified approach, J. Funct. Anal. 262 (2012), no. 1, 10-34. 
[21] Lutwak E., Yang D., Zhang G. L $L_{p}$ affine isoperimetric inequalities, J. Diff. Geom. 56 (2000), no. 1, 111-132.

[22] Lutwak E., Zhang G. Blaschke-Santaló inequalities, J. Diff. Geom. 47 (1997), no. $1,1-16$.

[23] Mankiewicz P., Tomczak-Jaegermann N. Quotients of finite-dimensional Banach spaces; random phenomena. In: "Handbook in the geometry of Banach spaces, Vol 2", ed. W. B. Johnson, J. Lindenstrauss, Elsevier, 1201-1246.

[24] Milman V.D., PAJor A. Isotropic positions and inertia ellipsoids and zonoids of the unit ball of a normed n-dimensional space. GAFA Seminar 87-89, Springer Lecture Notes in Math. 1376 (1989), 64-104.

[25] Milman V.D., Schechtman G. Asymptotic theory of finite-dimensional normed spaces. Lecture Notes in Math., 1200, Springer, Berlin-New York, 1985.

[26] Paouris G. Concentration of mass on convex bodies, Geometric and Functional Analysis 16 (2006), 1021-1049.

[27] Pisien G. Probabilistic methods in the geometry of Banach spaces, Probability and analysis (Varenna, 1985), Lecture Notes in Math., 1206 (1986), 167-241, Springer, Berlin.

David Alonso-Gutiérrez,

Dept. de Matemàtiques,

Universitat Jaume I,

Campus de Riu Sec.

E-12071. Castelló de la Plana. Spain.

e-mail: davidalonsogutierrez@gmail.com

Alexander E. Litvak,

Dept. of Math. and Stat. Sciences,

University of Alberta,

Edmonton, Alberta, Canada, T6G 2G1.

e-mail: aelitvak@gmail.com

Nicole Tomczak-Jaegermann,

Dept. of Math. and Stat. Sciences,

University of Alberta,

Edmonton, Alberta, Canada, T6G 2G1.

e-mail: nicole.tomczak@ualberta.ca 